\title{
THE IMPACT OF OMEGA-3 FATTY ACIDS COMBINED WITH INITIAL PERIODONTAL THERAPY ON SALIVARY VISFATIN AND TNF- $\alpha$ LEVELS IN CHRONIC PERIODONTITIS PATIENTS
}

\author{
Hesham M. El-Sharkawy* and Samah H. Elmeadawy*
}

\begin{abstract}
Recent investigations have demonstrated the positive therapeutic effects of omega-3 polyunsaturated fatty acids (PUFAs) on several chronic inflammatory diseases such as rheumatoid arthritis, chronic inflammatory bowel diseases and periodontitis. The objective of the present study was to evaluate the impact of omega-3 PUFAs in conjunction with initial periodontal therapy (IPT) on periodontal clinical parameters and salivary markers in patients with chronic periodontitis. Thirty four systemically healthy individuals with advanced chronic periodontitis were enrolled and randomly assigned into two groups. The control group (IPT, $n=17$ ) was treated with IPT alone whereas the test group (IPT+omega-3, $n=17$ ) was treated with IPT and daily dietary supplementation of omega-3 PUFAs (in the form of 2 grams of fish oil capsules per day) for three months. Clinical parameters were recorded at baseline and 3 months following therapy for both groups. Saliva samples were collected at the same time points and analyzed for visfatin and tumor necrosis factor- $\alpha$ (TNF- $\alpha$ ). After 3 months, clinical periodontal parameters of advanced chronic periodontitis were significantly improved in both groups. The omega-3 group showed significant greater pocket depth (PD) reduction and clinical attachment (CAL) gain compared to the control group after therapy. Salivary TNF- $\alpha$ levels showed a statistically significant decrease in the omega-3 group at 3 months compared to the control group. Salivary visfatin levels were reduced significantly at 3 months in both groups without any significant variation. It was concluded that dietary supplementation with omega-3 PUFAs could be a potential viable adjunct to IPT that significantly improves all periodontal parameters and reduces salivary visfatin and TNF- $\alpha$ levels in advanced chronic periodontitis. Interestingly, salivary visfatin could be a useful periodontal biomarker to monitor responses to periodontal therapy.
\end{abstract}

KEY WORDS: Omega-3 fatty acids, initial periodontal therapy, chronic periodontitis, visfatin, saliva.

*Associate Professor of Oral Medicine and Periodontology, Faculty of Dentistry, Mansoura University. 


\section{INTRODUCTION}

Chronic periodontitis is a local slowly progressing inflammatory disease affecting the periodontium and primarily initiated by specific anaerobic bacteria such as Porphyromonas gingivalis, Tanneralla forsythia and Treponema denticola which are collectively named "the red complex". (1) Most of the periodontal tissue destruction results from the exaggerated host response to infection and not by the direct effect of periodontopathogens. ${ }^{(2)}$ The host response to these pathogens result in the release of a bunch of pro-inflammatory cytokines such as tumor necrosis factor- $\alpha$ (TNF- $\alpha$ ), interleukin-1 (IL-1), IL-6 and prostaglandin-E2 (PGE2). The aforementioned cytokines could activate several matrix metalloproteinases (MMPs) which result in extracellular matrix degradation of the periodontal tissues and stimulate bone resorption. ${ }^{(3)}$

A variety of locally and systemically delivered pharmaceuticals have been assessed as host modulation therapeutics (HMTs) in the treatment of periodontal disease such as non-steroidal antiinflammatory drugs, tetracyclines, bisphosphonates and growth factors. ${ }^{(4)}$ To date, dietary supplementation of omega-3 polyunsaturated fatty acids (PUFAs) has emerged with positive effects on systemic inflammation and proposed as a promising adjunctive HMT for periodontal disease. ${ }^{(5)}$ Omega-3 PUFAs are naturally present in reasonably high concentrations in fish oil.(6) Two main classes have been described till now: eicosapentaenoic acid (EPA) and docosahexaenoic acid (DHA). It was revealed that both EPA and DHA have antiinflammatory effects in several diseases including periodontitis.(7) EPA and DHA are substrates for cyclooxygenase and lipoxygenase pathways with reverse actions compared to arachidonic acid, which is an omega-6 PUFA responsible for the synthesis of pro-inflammatory mediators. ${ }^{(8)}$ Based upon the structural similarity, there is a competition between omega-6 PUFA and omega-3 PUFA members balancing the anti- or pro-inflammatory function. If the equilibrium shifts in favor of omega-3 PUFAs, the synthesis of pro-inflammatory products including leukotriene- B4 (LT- B4) and PGE2 are down-regulated. ${ }^{(9)}$ Thus, omega-3 PUFAs could resolve inflammatory reactions via this mechanism. Omega-3 PUFAs are also substrates for generation of a novel series of bioactive lipid mediators known as resolvins and protectins that show potent protective actions in several inflammatory diseases including periodontitis. ${ }^{(10)}$ The effects of omega-3 PUFAs have been investigated in a variety of experimental animal models.(11) In humans, dietary supplementation with omega-3 PUFAs has the likelihood to yield a considerable clinical outcome and pharmacologically interfere with the inflammatory cascade in saliva. ${ }^{(12)}$ However, limited few data suggest that omega-3 PUFAs may have the potential as a host modulatory therapeutic for the prevention and/or adjuvant of periodontal disease management. ${ }^{(13)}$

Recently, visfatin was discovered as a novel adipocytokine produced mainly in the visceral adipose tissues and has insulin-like functions. ${ }^{(14)}$ In human studies, visfatin was also detected in blood cells such as monocytes ${ }^{(15)}$, lymphocytes ${ }^{(16)}$, macrophages ${ }^{(17,18)}$ as well as dendritic cells ${ }^{(17}$. It has a pro-inflammatory function and secreted by polymorphonuclear leukocytes (PMNs) in response to inflammatory process. ${ }^{(19)}$ Visfatin stimulates the release of pro-inflammatory cytokines from monocytes ${ }^{(17)}$ suggesting a possible role in the development of inflammatory diseases. Visfatin might play a role in the regulation of defense and immune functions. ${ }^{(19)}$ Furthermore, visfatin molecule is over-expressed in several inflammatory disorders such as acute lung injury, rheumatoid arthritis, psoriasis and inflammatory bowel diseases. ${ }^{(20-23)}$ It has a principal role in the development of chronic inflammatory foci by inhibiting PMNs apoptosis. ${ }^{(21)}$ Periodontal inflammation up-regulates several pro-inflammatory cytokines which eventually lead to over-ex- 
pression of visfatin in periodontal tissues. ${ }^{(24)}$ It was also revealed that visfatin concentrations in serum and gingival crevicular fluid (GCF) in periodontal health and disease were quantified suggesting correlated increased visfatin concentrations in serum and GCF with severity of periodontitis. ${ }^{(25)}$

Nowadays, an increased interest in saliva as a diagnostic fluid was increased among researchers. In the field of periodontics, saliva is reported to reflect the soluble mediator composition of the periodontal tissues, GCF and saliva of subjects suffering from periodontal diseases. ${ }^{(26)}$ Thus, saliva contains specific biomarkers for the unique physiopathological aspects of periodontal disease, and alteration in the concentrations of these biomarkers plays an important role in diagnostic and therapeutic approaches. $^{(27)}$

Hence, in the current study, we hypothesized that dietary supplementation of omega-3 PUFAs would improve the clinical outcomes of periodontal treatment and reduce salivary inflammatory markers associated with disease. Therefore, we evaluated the impact of omega-3 PUFAs in conjunction with initial periodontal therapy (IPT) on the changes in standard periodontal measurements and salivary concentrations of both visfatin and TNF- $\alpha$ in patients with advanced chronic periodontitis.

\section{MATERIALS AND METHODS}

\section{Study Population}

Thirty four (34) subjects were enrolled in the study from the Periodontology Clinic at the Faculty of Dentistry, Mansoura University, Egypt, between February and August, 2016. An approval for the study was obtained from Research Ethics Committee in the Faculty of Dentistry. The inclusion criteria included good systemic health (by questionnaire), untreated advanced chronic periodontitis, age 35 to 60 years, and the presence of $\geq 18$ teeth (excluding third molars and teeth with fixed restorations or dental implants). Advanced chronic periodontitis was defined as the presence of at least 6 teeth with a probing depth (PD) $>6 \mathrm{~mm}$, clinical attachment loss (CAL) $\geq 4 \mathrm{~mm}$ and evident alveolar bone loss as detected in the radiographs. Exclusion criteria included presence of any systemic disease (i.e., diabetes mellitus), cancer, radiotherapy, immunosuppressive or corticosteroid therapy, smoking, pregnant or lactating women, any antibiotic taken within the previous 2 months, current use of non-steroidal anti-inflammatory drugs, and any form of previous periodontal treatment.

After clarification of all aspects of the study, the selected patients signed an informed consent for participation in the study and each one was assigned a study number in an ascending order. Enrolled patients were subjected to initial periodontal therapy (IPT) which included full mouth thorough scaling and root planing (SRP), restoration of carious lesions and food impaction areas, extraction of hopeless teeth and giving strict oral hygiene instructions for plaque control. No antibiotics were prescribed for patients during the whole study period. After completion of IPT phase, subjects were divided randomly into two groups:

Group I (IPT): consisted 17 patients treated only with initial periodontal therapy (IPT) to act as control group.

Group II (IPT+Omega-3): included 17 patients treated with IPT followed by dietary supplementation with two grams of fish oil (Omega 3 Plus $^{\mathrm{TM}}$ capsules, produced by SEDICO Pharmaceutical Co., 6 October City-Egypt) divided twice daily for three months. Each Omega 3 Plus capsule contains $1000 \mathrm{mg}$ fish oil (EPA/DHA 30\%) and $100 \mathrm{mg}$ wheat germ oil.

After taking medical and dental histories from enrolled individuals, complete thorough clinical examination was performed and a comprehensive treatment plan was scheduled. IPT was performed 
for all enrolled patients and included full mouth SRP by hand and ultrasonic instruments in addition to giving oral hygiene instructions. SRP was performed by the same periodontist (SE).

At baseline (i.e. before commencement of initial periodontal therapy) and after 3 months of therapy, clinical measurements including Plaque Index (Turesky modification of Quigley-Hein, PI) ${ }^{28}$, Modified Gingival Index (MGI) ${ }^{29}$, bleeding on probing (BOP) scores (dichotomous), probing pocket depth (PD) and clinical attachment level (CAL) were measured. Subjects came to the clinic every month during the course of the study (3 months) to replenish their medication. Remaining medications were quantified as a measure of compliance. At each evaluation visit (monthly), all patients were evaluated for plaque control and examined for detection of the presence of any oral soft and hard tissue abnormalities. Moreover, patients in group II were asked about any adverse reactions due to medication intake.

\section{Saliva sampling and analysis}

At baseline and 3 months, whole unstimulated saliva samples $(5 \mathrm{ml})$ samples were collected by asking the patients to expectorate into polypropylene tubes before measuring the clinical parameters. The saliva samples were centrifuged to remove debris and immediately frozen at $-70^{\circ} \mathrm{C}$ until all samples were collected. Salivary visfatin and TNF- $\alpha$ levels were quantified by ELISA. The human visfatin and TNF- $\alpha$ ELISA kits (R\&D systems, Minneapolis, USA) were utilized to quantify these analytes in saliva samples according to the manufacturers' recommendations. Salivary concentrations of both visfatin and TNF- $\alpha$ assays were expressed as $\mathrm{ng} / \mathrm{ml}$ and $\mathrm{pg} / \mathrm{ml}$ concentrations, respectively.

\section{Statistics}

The study power was performed with type I error $\alpha=0.05, \beta=0.17$ and $1-\beta=0.83$. The power was calculated as $\mathrm{p}=0.8332$ and the estimated minimum sample size required was 15 per each group to achieve a power of $80 \%$. The whole study data were explored for normality by using Kolmogrov-Smirnov test. Parametric data were presented as mean \pm SD except for salivary visfatin and TNF- $\alpha$ levels which were represented as mean \pm SEM (standard error of the mean). Demographic variables and baseline data were compared by unpaired t-test. Student $t$ test was used for the data obtained at baseline and at 3 months after therapy, to determine whether there were significant differences between treatment groups. Data were analysed with the Statistical Package for Social Science (SPSS) program, version 19 . The significance limit was set at $5 \%$.

\section{RESULTS}

The demographic features regarding age, age range and sex in test and control groups are shown in Table1. The aforementioned features exhibited no significant difference between groups. The same table also demonstrated that there were no statistically significant variation between the two groups regarding the average values $( \pm \mathrm{SD})$ of body mass index (BMI), number of teeth, plaque index (PI), modified gingival index (MGI), bleeding on probing score (BOP), probeable pocket depth (PD) and clinical attachment loss (CAL) as well as the mean $( \pm$ SEM $)$ of salivary levels of both visfatin and TNF- $\alpha$ measured as $\mathrm{ng} / \mathrm{ml}$ and $\mathrm{pg} / \mathrm{ml}$, respectively ( $p>0.05)$. All patients returned for scheduled maintenance and follow-up visits and there were no dropouts. No abnormalities were observed in oral soft and hard tissue examinations. Patients did not report any gastrointestinal disorders or other adverse events due to intake of omega-3 capsules.

The full mouth mean values of both groups regarding periodontal measurements at baseline and at 3 months after therapy are exhibited in Table 2. The PI reduction for the IPT and omega- 3 groups at 3 month showed non-significant variations. 
Moreover, there was no statistically significant difference between the two groups regarding MGI and BOP after therapy (P>0.05). However, PD and $\mathrm{CAL}$ in the omega-3 group were significantly improved compared to the control values at 3 months $(\mathrm{P}<0.001)$. The mean values of pocket depth reduction and clinical attachment gain were calculated in control and omega-3 groups after 3 months as shown in Fig.1A and B. The mean PD reductions in the control and omega-3 groups were $1.09 \pm 0.6 \mathrm{~mm}$ and $2.52 \pm 0.55 \mathrm{~mm}$, respectively; with statistically significant difference, $\mathrm{p}<0.001$ (Fig.1A). In the same context, the mean CAL gain in the control and omega-3 groups were $1.24 \pm 0.47 \mathrm{~mm}$ and $2.38 \pm 0.44 \mathrm{~mm}$, respectively; with statistically significant variation, $\mathrm{p}<0.001$ (Fig.1B).

The mean $( \pm$ SEM) values of salivary visfatin levels of both groups at baseline and after therapy are shown in Figure 2A. The mean values of salivary visfatin levels were $33.42 \pm 2.42 \mathrm{ng} / \mathrm{ml}$ in the omega- 3 group and $32.27 \pm 3.25 \mathrm{ng} / \mathrm{ml}$ in the control group at baseline $(\mathrm{P}>0.05)$. After therapy, both omega-3 and control group showed a significant reduction in visfatin concentrations (reduced to $18.78 \pm 2.17$ and $17.51 \pm 2.84 \mathrm{ng} / \mathrm{ml}$, respectively) when compared to baseline values $(\mathrm{P}<0.001)$. However, no significant variation of visfatin levels was noted between groups at 3- month values ( $\mathrm{P}>0.05)$.

Furthermore, the mean $( \pm$ SEM $)$ values of salivary TNF- $\alpha$ levels of both groups at baseline and after therapy are shown in Figure 2B. The mean values of salivary TNF- $\alpha$ levels were $12.67 \pm 1.12 \mathrm{pg} / \mathrm{ml}$ in the omega- 3 group and $13.61 \pm 1.14 \mathrm{pg} / \mathrm{ml}$ in the control group at baseline $(\mathrm{P}>0.05)$. Both groups demonstrated significant reduction with regard to baseline at 3 months. At 3 months, the mean value of TNF- $\alpha$ level was $6.72 \pm 1.17 \mathrm{pg} / \mathrm{ml}$ in the omega-3 group and $8.97 \pm 1.09 \mathrm{pg} / \mathrm{ml}$ in the control group, and these values were statistically significant compared to baseline values $(\mathrm{P}<0.001)$ and to each other $(\mathrm{P}<0.01)$.

TABLE (1) Demographic Features and Baseline Data of Study Groups

\begin{tabular}{|l|c|c|}
\hline Variable & IPT $(\mathbf{n = 1 7})$ & IPT+Omega-3 (n=17) \\
\hline Age, year $(\mathbf{M} \pm$ SD) & $47.82 \pm 2.21$ & $40.75 \pm 2.05$ \\
\hline Age range, year (min.-max.) & $41-50$ & $8(47)$ \\
\hline Female, $\mathbf{( \% )}$ & $9(53)$ & $26.1 \pm 1.2$ \\
\hline BMI $\left(\mathbf{K g} / \mathbf{m}^{2)}\right.$ & $25.3 \pm 1.4$ & $24.1 \pm 3.2$ \\
\hline No. of teeth & $25.1 \pm 2.1$ & $2.43 \pm 0.36$ \\
\hline PI score & $2.38 \pm 0.76$ & $2.32 \pm 0.19$ \\
\hline MGI score & $2.27 \pm 0.13$ & $0.91 \pm 0.25$ \\
\hline BOP score & $0.88 \pm 0.09$ & $4.76 \pm 0.84$ \\
\hline PD $(\mathbf{m m})$ & $4.46 \pm 0.57$ & $5.16 \pm 0.52$ \\
\hline CAL $(\mathbf{m m})$ & $5.08 \pm 0.46$ & $32.27 \pm 3.25$ \\
\hline Salivary Visfatin $(\mathbf{n g} / \mathbf{m l})(\mathbf{M} \mathbf{S E M})$ & $33.42 \pm 2.42$ & $12.76 \pm 2.51$ \\
\hline Salivary TNF- $\boldsymbol{\alpha}(\mathbf{p g} / \mathbf{m l})(\mathbf{M} \pm$ SEM) & $13.61 \pm 3.14$ & \\
\hline
\end{tabular}

p-value is not statistically significant (Unpaired t-test; $p>0.05$ ). 


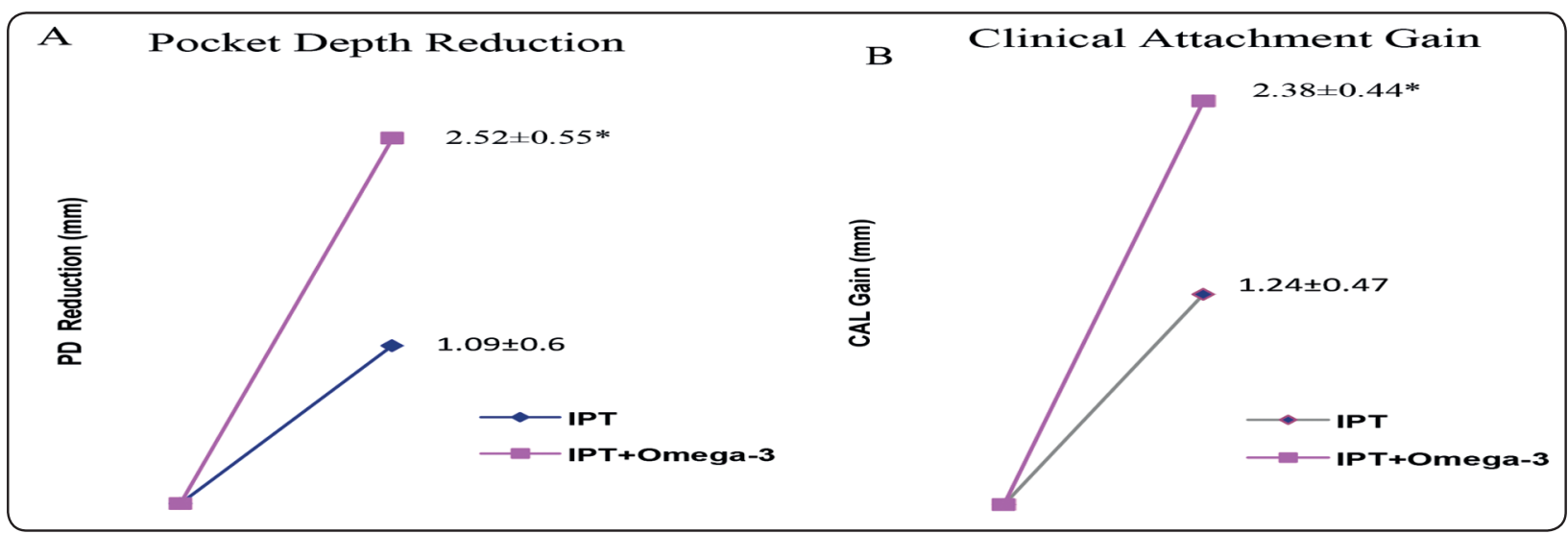

Fig. (1) Panels A and B exhibited the mean $( \pm \mathrm{SD})$ values of full mouth pocket depth (PD) reduction and clinical attachment (CAL) gain which were significantly greater in IPT+Omega-3 group compared to IPT group after therapy; Student t test; $*$ p $<0.001$.

TABLE (2) Periodontal Measurements of Study Groups at Baseline and After 3 Months of Therapy

\begin{tabular}{|l|c|c|c|c|}
\hline \multirow{2}{*}{ Variable } & \multicolumn{2}{|c|}{ IPT $(\mathbf{n = 1 7})$} & \multicolumn{2}{c|}{ IPT+Omega-3 (n=17) } \\
\cline { 2 - 5 } & baseline & after 3 M. & baseline & after 3 M. \\
\hline PI score & $2.38 \pm 0.76$ & $0.86 \pm 0.47^{*}$ & $2.43 \pm 0.36$ & $0.79 \pm 0.35^{*}$ \\
\hline MGI score & $2.27 \pm 0.13$ & $0.72 \pm 0.14^{*}$ & $2.32 \pm 0.19$ & $0.66 \pm 0.16^{*}$ \\
\hline BOP & $0.88 \pm 0.13$ & $0.14 \pm 0.09^{*}$ & $0.91 \pm 0.15$ & $0.12 \pm 0.06^{*}$ \\
\hline PD $(\mathbf{m m})$ & $4.46 \pm 0.57$ & $3.37 \pm 0.64^{*}$ & $4.76 \pm 0.84$ & $2.24 \pm 0.27 * \#$ \\
\hline CAL $(\mathbf{m m})$ & $5.08 \pm 0.46$ & $3.84 \pm 0.49^{*}$ & $5.16 \pm 0.52$ & $2.78 \pm 0.38^{*} \#$ \\
\hline
\end{tabular}

* p-value is statistically significant compared to baseline values (Student t-test; $p<0.001$ ).

\# p-value is statistically significant compared to 3 months control values (Student t-test; $p<0.001$ ).

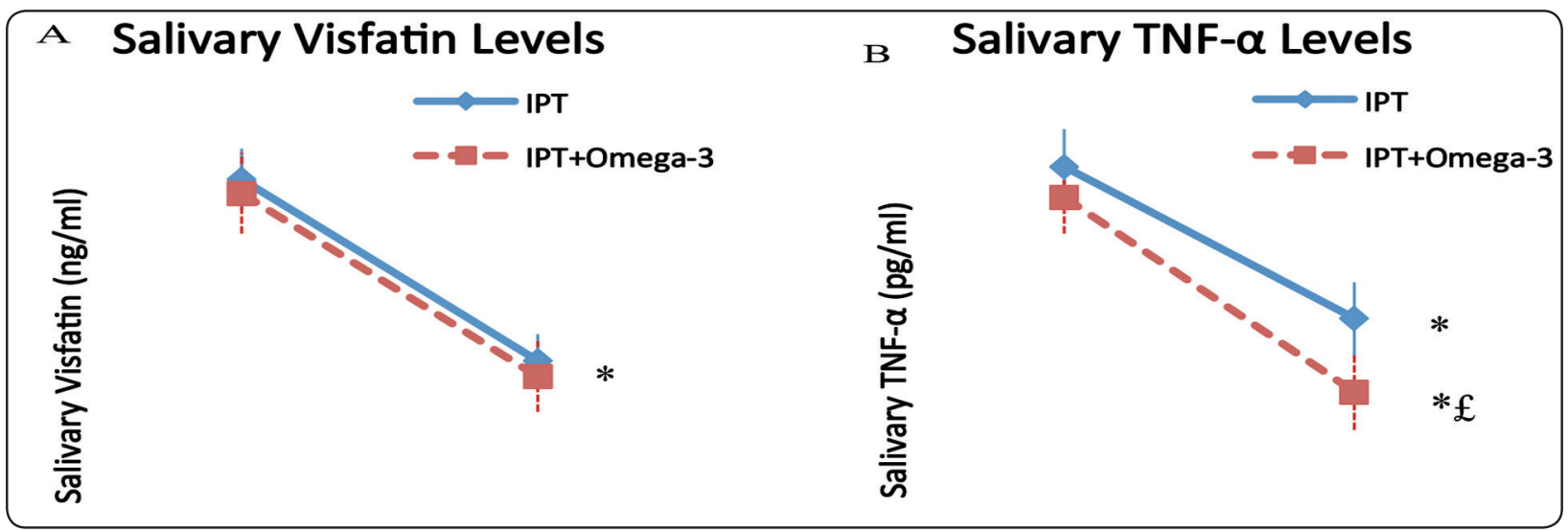

Fig. (1) Panels A and B exhibited the mean $( \pm \mathrm{SD})$ values of full mouth pocket depth $(\mathrm{PD})$ reduction and clinical attachment $(\mathrm{CAL})$ gain which were significantly greater in IPT+Omega-3 group compared to IPT group after therapy; Student $\mathrm{t}$ test; ${ }^{*} \mathrm{p}<0.001$. 


\section{DISCUSSION}

There is a large body of evidence indicating that omega-3 PUFAs are important viable agents in controlling inflammation in several inflammatory diseases. ${ }^{(30-32)}$ Nevertheless, it is not completely understood how omega-3 PUFAs affect periodontal disease outcomes.(12) The objective of our study was to determine whether the adjunctive use of omega-3 PUFAs oral supplementation in chronic periodontitis patients would have an impact on clinical parameters and salivary markers of periodontal disease. In total, our results showed that adjunctive omega-3 PUFAs supplementation with IPT significantly improved clinical and salivary biochemical outcomes in comparison to IPT alone. In our study, all the clinical parameters showed significant improvements at 3 months compared to baseline in both groups and this comes in accordance with previous investigations..$^{(12,33,34)}$ Similar to our study groups, the effect of dietary supplementation of omega-3 PUFAs with SRP on clinical periodontal indices in chronic periodontitis patients was investigated in two studies, which showed contrary results. The first study (35) demonstrated that dietary supplementation of omega-3 PUFAs with initial periodontal therapy showed no impact on clinical parameters while the second reported the opposite. ${ }^{(33)}$ Unlike our study groups, Rosenstein and his colleagues revealed no effect of omega-3 PUFAs on gingival index and probing depth without any periodontal intervention in periodontitis patients. ${ }^{(36)}$ Another study showed no improvement in the clinical parameters of individuals with experimental gingivitis. ${ }^{(37)}$

However, in the present study, we found positive effect of omega-3 PUFAs on PD reduction and CAL gain when compared to the control group. This could be explained because we have used 2 grams of fish oil per day that contain approximately $600 \mathrm{mg}$ of omega-3 PUFAs, however, the aforementioned studies used less amount of omega-3 PUFAs. Although PI, GI and BOP scores showed significant reductions after therapy in both omega-3 and control groups, they were not statistically significant compared to each other. Perhaps, this occurred because the effect of omega-3 PUFAs was too subtle to detect with the current small sample size.

In the present study, we have tested salivary visfatin as a new biomarker in evaluating the response of periodontal disease to IPT alone compared to adjunctive omega-3 PUFAs with IPT. In addition, we also assessed salivary TNF- $\alpha$ levels because it is an established salivary marker for periodontal disease as reported in previous studies. ${ }^{(38,39)}$ A limited number of studies have used visfatin as a potential periodontal marker in saliva, serum and gingival crevicular fluid (GCF) demonstrating that it could be a new reliable periodontal biomarker. ${ }^{(40-}$ 42) It was reported that increased visfatin expression has been associated with a variety of inflammatory diseases. ${ }^{(43,44)}$ Visfatin stimulates the release of proinflammatory cytokines such as TNF- $\alpha$ and IL-8 by peripheral mononuclear cells. ${ }^{(45)}$ Thus, it could be hypothesized that there is a strong link between visfatin and the pathogenesis of periodontitis. It was indicated that visfatin concentration in serum and GCF would be a possible marker for inflammatory activity in periodontitis. ${ }^{(46)}$ In the same context, our results demonstrated that salivary visfatin levels decreased significantly in patients with severe chronic periodontitis in response to both adjunctive omega-3 supplementation with IPT and IPT alone after 3 months compared to baseline values. Previous investigations have demonstrated reductions in the salivary levels of a variety of enzymes and cytokines in response to non-surgical periodontal therapy. ${ }^{(47,48)}$

TNF- $\alpha$ is a proinflammatory and immunoregulatory cytokine which is crucial in the pathogenesis of numerous inflammatory diseases and can be detected in saliva in both healthy periodontium and periodontal disease. ${ }^{(49)}$ It was revealed that the increased TNF- $\alpha$ concentration observed in periodontitis correlate strongly with the severity of tissue destruction. ${ }^{(50)}$ In our study, significant reductions in TNF- $\alpha$ levels in saliva occurred in both groups at 3 months as compared 
with baseline indicating that selected salivary biomarkers reflected periodontal status and response to periodontal therapy over time in all participants. This result is consistent with Sexton et al., who evaluated salivary TNF- $\alpha$ levels after SRP. ${ }^{(51)}$ Most importantly, in our study, salivary TNF- $\alpha$ levels were significantly lower in omega-3 PUFAs group compared to control group at 3 months. Accordingly, adjunctive use of omega-3 PUFAs oral supplementation for 3 months by subjects treated for chronic periodontitis could be sufficient to resolve the inflammatory state of chronic periodontitis patients. In our study, we did not evaluate the TNF- $\alpha$ levels in serum; however, we might hypothesize that the significant decrease in salivary TNF- $\alpha$ due to omega-3 PUFAs intake could be associated with the systemic reduction of serum TNF- $\alpha$ levels. It could be explained as increasing the intake of omega-3 PUFAs can modify the fatty-acid composition of cells and this occurs in a dose-response over an interval of weeks to months. Based on these findings, we could speculate that the significant effect of omega-3 PUFAs on TNF- $\alpha$ levels only at 3 months may be associated with a time-dependent cumulative effect.

In our study, we evaluated visfatin and TNF- $\alpha$ levels in saliva because it is readily available and easily collected without any need for certain tools or exerting more effort by clinicians. Moreover, various mediators of periodontal tissue destruction and inflammation have been detected in the whole saliva of periodontitis patients previously. ${ }^{(52)}$ The unstimulated saliva contains biological materials collected from all periodontal sites and assessment of salivary markers provide an overall image of disease status other than site-specific GCF analysis. In addition, salivary biomarkers in periodontal disease have been investigated mostly in cross-sectional studies, however, the number of available longitudinal studies is limited. ${ }^{(38,39,53)}$ Thus, a 6-month longitudinal study to follow chronic periodontitis patients who received SRP with/ without omega-3 PUFAs supplementation may help to understand the effects of these markers for followup assessment. Limited data suggest that the dietary omega-3 PUFAs could be effective in improving resistance to extracellular pathogens. ${ }^{(11)}$ However, the antimicrobial activity of omega-3 PUFAs on the subgingival pathogens in naturally occurring periodontitis have not been thoroughly studied and presents an area of interest for modifying the host response to the disease.

\section{CONCLUSIONS}

1- This study suggests that daily dietary supplementation with omega-3 PUFAs could be a promising sustainable adjunctive host modulation therapeutic for periodontal disease.

2- The combination of omega-3 PUFAs to IPT can reduce salivary TNF- $\alpha$ levels in advanced chronic periodontitis patients more than IPT alone.

3- IPT alone or together with omega-3 PUFAs have the ability to significantly decrease salivary visfatin concentrations in severe chronic periodontitis patients.

4- Salivary visfatin might be useful for monitoring responses to periodontal therapy. However, for visfatin to be considered as a potential periodontal biomarker in the treatment of periodontal disease, further longitudinal studies with larger sample sizes are warranted.

\section{REFERNCES}

1. Holt SC, Ebersole JL. Porphyromonas gingivalis, Treponema denticola, and Tannerella forsythia: the "red complex", a prototype polybacterial pathogenic consortium in periodontitis. Periodontology 2000 2005;38:72-122.

2. Huang N, Gibson FC, 3rd. Immuno-pathogenesis of Periodontal Disease: Current and Emerging Paradigms. Current oral health reports 2014;1:124-132.

3. Cekici A, Kantarci A, Hasturk H, Van Dyke TE. Inflammatory and immune pathways in the pathogenesis of periodontal disease. Periodontology 2000 2014; 64:57-80. 
4. Salvi GE, Lang NP. Host response modulation in the management of periodontal diseases. Journal of clinical periodontology 2005;32 Suppl 6:108-129.

5. Schubert R, Kitz R, Beermann C, et al. Influence of lowdose polyunsaturated fatty acids supplementation on the inflammatory response of healthy adults. Nutrition 2007;23:724-730.

6. Kaye EK. n-3 fatty acid intake and periodontal disease. Journal of the American Dietetic Association 2010; 110:1650-1652.

7. Serhan CN, Chiang N, Van Dyke TE. Resolving inflammation: dual anti-inflammatory and pro-resolution lipid mediators. Nature reviews Immunology 2008; 8:349-361.

8. Healy DA, Wallace FA, Miles EA, Calder PC, Newsholm P. Effect of low-to-moderate amounts of dietary fish oil on neutrophil lipid composition and function. Lipids 2000; 35:763-768.

9. Calder PC. Polyunsaturated fatty acids and inflammation. Prostaglandins, leukotrienes, and essential fatty acids 2006;75:197-202.

10. Hasturk H, Kantarci A, Van Dyke TE. Paradigm shift in the pharmacological management of periodontal diseases. Frontiers of oral biology 2012;15:160-176.

11. Dawson DR, 3rd, Branch-Mays G, Gonzalez OA, Ebersole JL. Dietary modulation of the inflammatory cascade. Periodontology 2000 2014;64:161-197.

12. El-Sharkawy H, Aboelsaad N, Eliwa M, et al. Adjunctive treatment of chronic periodontitis with daily dietary supplementation with omega-3 Fatty acids and low-dose aspirin. Journal of periodontology 2010;81:1635-1643.

13. Chee B, Park B, Fitzsimmons T, Coates AM, Bartold PM. Omega-3 fatty acids as an adjunct for periodontal therapy-a review. Clinical oral investigations 2016;20:879-894.

14. Fukuhara A, Matsuda M, Nishizawa M, et al. Visfatin: a protein secreted by visceral fat that mimics the effects of insulin. Science 2005;307:426-430.

15. Dahl TB, Yndestad A, Skjelland M, et al. Increased expression of visfatin in macrophages of human unstable carotid and coronary atherosclerosis: possible role in inflammation and plaque destabilization. Circulation 2007;115:972-980.

16. Samal B, Sun Y, Stearns G, Xie C, Suggs S, McNiece I. Cloning and characterization of the cDNA encoding a novel human pre-B-cell colony-enhancing factor. Molecular and cellular biology 1994;14:1431-1437.

17. Moschen AR, Kaser A, Enrich B, et al. Visfatin, an adipocytokine with proinflammatory and immunomodulating properties. Journal of immunology 2007; 178:1748-1758.

18. Curat CA, Wegner V, Sengenes C, et al. Macrophages in human visceral adipose tissue: increased accumulation in obesity and a source of resistin and visfatin. Diabetologia 2006;49:744-747.

19. Sethi JK, Vidal-Puig A. Visfatin: the missing link between intra-abdominal obesity and diabetes? Trends in molecular medicine 2005;11:344-347.

20. Nowell MA, Richards PJ, Fielding CA, et al. Regulation of pre-B cell colony-enhancing factor by STAT-3dependent interleukin-6 trans-signaling: implications in the pathogenesis of rheumatoid arthritis. Arthritis and rheumatism 2006;54:2084-2095.

21. Jia SH, Li Y, Parodo J, et al. Pre-B cell colony-enhancing factor inhibits neutrophil apoptosis in experimental inflammation and clinical sepsis. The Journal of clinical investigation 2004;113:1318-1327.

22. Ye SQ, Simon BA, Maloney JP, et al. Pre-B-cell colonyenhancing factor as a potential novel biomarker in acute lung injury. American journal of respiratory and critical care medicine 2005;171:361-370.

23. Koczan D, Guthke R, Thiesen HJ, et al. Gene expression profiling of peripheral blood mononuclear leukocytes from psoriasis patients identifies new immune regulatory molecules. European journal of dermatology : EJD 2005; 15:251-257.

24. Ognjanovic S, Bao S, Yamamoto SY, Garibay-Tupas J, Samal B, Bryant-Greenwood GD. Genomic organization of the gene coding for human pre-B-cell colony enhancing factor and expression in human fetal membranes. Journal of molecular endocrinology 2001;26:107-117.

25. Pradeep AR, Raghavendra NM, Prasad MV, Kathariya R, Patel SP, Sharma A. Gingival crevicular fluid and serum visfatin concentration: their relationship in periodontal health and disease. Journal of periodontology 2011;82:1314-1319.

26. Taylor JJ. Protein biomarkers of periodontitis in saliva. ISRN inflammation 2014;2014:593151.

27. AlMoharib HS, AlMubarak A, AlRowis R, Geevarghese A, Preethanath RS, Anil S. Oral fluid based biomarkers in 
periodontal disease: part 1. Saliva. Journal of international oral health : JIOH 2014;6:95-103.

28. Turesky S, Gilmore ND, Glickman I. Reduced plaque formation by the chloromethyl analogue of victamine C. Journal of periodontology 1970;41:41-43.

29. Gordon JM, Lamster IB, Seiger MC. Efficacy of Listerine antiseptic in inhibiting the development of plaque and gingivitis. Journal of clinical periodontology 1985;12:697704

30. Kitz R, Rose MA, Schubert R, et al. Omega-3 polyunsaturated fatty acids and bronchial inflammation in grass pollen allergy after allergen challenge. Respiratory medicine 2010;104:1793-1798.

31. Kooshki A, Taleban FA, Tabibi H, Hedayati M. Effects of marine omega-3 fatty acids on serum systemic and vascular inflammation markers and oxidative stress in hemodialysis patients. Annals of nutrition \& metabolism 2011;58:197-202.

32. Tull SP, Yates CM, Maskrey BH, et al. Omega-3 Fatty acids and inflammation: novel interactions reveal a new step in neutrophil recruitment. PLoS biology 2009;7:e1000177.

33. Deore GD, Gurav AN, Patil R, Shete AR, Naiktari RS, Inamdar SP. Omega 3 fatty acids as a host modulator in chronic periodontitis patients: a randomised, double-blind, palcebo-controlled, clinical trial. Journal of periodontal \& implant science 2014;44:25-32.

34. Elwakeel NM, Hazaa HH. Effect of omega 3 fatty acids plus low-dose aspirin on both clinical and biochemical profiles of patients with chronic periodontitis and type 2 diabetes: a randomized double blind placebo-controlled study. Journal of periodontal research 2015;50:721-729.

35. Martinez GL, Koury JC, Brito F, Fischer RG, Gustafsson A, Figueredo CM. The impact of non-surgical periodontal treatment on serum levels of long chain-polyunsaturated fatty acids: a pilot randomized clinical trial. Journal of periodontal research 2014;49:268-274.

36. Rosenstein ED, Kushner LJ, Kramer N, Kazandjian G. Pilot study of dietary fatty acid supplementation in the treatment of adult periodontitis. Prostaglandins, leukotrienes, and essential fatty acids 2003;68:213-218.

37. Campan P, Planchand PO, Duran D. Pilot study on n-3 polyunsaturated fatty acids in the treatment of human experimental gingivitis. Journal of clinical periodontology 1997;24:907-913.
38. Nomura Y, Shimada Y, Hanada N, et al. Salivary biomarkers for predicting the progression of chronic periodontitis. Archives of oral biology 2012;57:413-420.

39. Salminen A, Gursoy UK, Paju S, et al. Salivary biomarkers of bacterial burden, inflammatory response, and tissue destruction in periodontitis. Journal of clinical periodontology 2014;41:442-450.

40. Tabari ZA, Ghaedi FB, Azadmehr A, et al. Salivary Visfatin Concentration in Response to Non-surgical Periodontal Therapy. Journal of clinical and diagnostic research : JCDR 2015;9:ZC05-08.

41. Mishra V, Shettar L, Bajaj M, Math AS, Thakur SL. Interlinking Periodontitis and Type 2 Diabetes Mellitus by Assessment of Crevicular Visfatin Levels in Health and in Disease Before and After Initial Periodontal Therapy. Journal of clinical and diagnostic research : JCDR 2016;10:ZC67-71.

42. Raghavendra NM, Pradeep AR, Kathariya R, Sharma A, Rao NS, Naik SB. Effect of non surgical periodontal therapy on gingival crevicular fluid and serum visfatin concentration in periodontal health and disease. Disease markers 2012;32:383-388.

43. Varma V, Yao-Borengasser A, Rasouli N, et al. Human visfatin expression: relationship to insulin sensitivity, intramyocellular lipids, and inflammation. The Journal of clinical endocrinology and metabolism 2007;92:666-672.

44. Juan X, Lu YM, Shi JD, Deng XQ, Long W. Visfatin levels in patients with severe pneumonia. World journal of emergency medicine 2011;2:132-136.

45. Zhang J, Zhou L, Tang L, Xu L. Plasma visfatin levels and mRNA expression of visfatin in peripheral blood mononuclear cells and peripheral blood monocyte-derived macrophages from normal weight females with polycystic ovary syndrome. Experimental and therapeutic medicine 2014;7:1215-1220.

46. Wu Y, Chen L, Wei B, Luo K, Yan F. Effect of non-surgical periodontal treatment on visfatin concentrations in serum and gingival crevicular fluid of patients with chronic periodontitis and type 2 diabetes mellitus. Journal of periodontology 2015;86:795-800.

47. Aurer A, Stavljenic-Rukavina A, Aurer-Kozelj J. [Markers of periodontal destruction in saliva of periodontitis patients]. Acta medica Croatica : casopis Hravatske akademije medicinskih znanosti 2005;59:117-122. 
48. Ingman T, Sorsa T, Lindy O, Koski H, Konttinen YT. Multiple forms of gelatinases/type IV collagenases in saliva and gingival crevicular fluid of periodontitis patients. Journal of clinical periodontology 1994;21:26-31.

49. Ide M, Jagdev D, Coward PY, Crook M, Barclay GR, Wilson RF. The short-term effects of treatment of chronic periodontitis on circulating levels of endotoxin, C-reactive protein, tumor necrosis factor-alpha, and interleukin-6. Journal of periodontology 2004;75:420-428.

50. Gursoy UK, Yildiz Ciftlikli S, Kononen E, Gursoy M, Dogan B. Salivary interleukin-17 and tumor necrosis factoralpha in relation to periodontitis and glycemic status in type 2 diabetes mellitus. Journal of diabetes 2015;7:681-688.
51. Sexton WM, Lin Y, Kryscio RJ, Dawson DR, 3rd, Ebersole JL, Miller CS. Salivary biomarkers of periodontal disease in response to treatment. Journal of clinical periodontology 2011;38:434-441.

52. Varghese SS, Thomas H, Jayakumar ND, Sankari M, Lakshmanan R. Estimation of salivary tumor necrosis factor-alpha in chronic and aggressive periodontitis patients. Contemporary clinical dentistry 2015; 6:S152-156.

53. Kibayashi M, Tanaka M, Nishida N, et al. Longitudinal study of the association between smoking as a periodontitis risk and salivary biomarkers related to periodontitis. Journal of periodontology 2007;78:859-867. 